\title{
Symptomatic Efficacy of Pharmacological Treatments for Knee Osteoarthritis: A Systematic Review and a Network Meta-Analysis with a 6-Month Time Horizon
}

\author{
C. Beaudart ${ }^{1}$ - $\cdot$ L. Lengelé ${ }^{1} \cdot$ V. Leclercq ${ }^{1} \cdot$ A. Geerinck ${ }^{1} \cdot$ D. Sanchez-Rodriguez ${ }^{1,2} \cdot$ O. Bruyère $^{1} \cdot$ J. Y. Reginster ${ }^{1,3}$
}

Published online: 19 October 2020

(c) The Author(s) 2020

\begin{abstract}
Introduction Several pharmacological treatments aiming at a better symptomatic control of osteoarthritis (OA) are used in daily practice but their efficacy is often disputed. The purpose of this network meta-analysis (NMA) is to assess the efficacy on pain and function of the drugs that are most widely prescribed against knee OA.

Methods Medline, Scopus, and Cochrane database of systematic reviews were searched for randomized controlled trials published up to August 2019 and assessing the efficacy of knee OA treatments using a 6-month time horizon. Pain and function changes from baseline were the primary outcomes. A Bayesian network meta-analysis was run and standardized mean differences (SMDs) with 95\% credibility intervals (95\% CrIs) were calculated.

Results 9697 references were identified and 80 RCTs were concordant with our inclusion criteria (79 studies involving 15,609 individuals reported pain outcomes and 55 studies involving 13,655 individuals reported function outcomes). A significant decrease in pain was observed for the intra-articular (IA) combination of hyaluronic acid (HA) and triamcinolone (SMD - 0.49, 95\% CrI - 0.78; - 0.19), vitamin D (SMD - 0.31, 95\% CrI - 0.56; - 0.06), IA HA (SMD - 0.29, 95\% CrI $-0.40 ;-0.17$ ), prescription-grade crystalline glucosamine sulfate (pCGS) (SMD $-0.29,95 \%$ CrI $-0.58 ;-0.004$ ), and prescription-grade chondroitin sulfate (pCS) (SMD - 0.26, 95\% CrI - 0.44; - 0.08). Significant improvements in physical function were found with pCGS (SMD - 0.44, 95\% CrI - 0.66; - 0.21), vitamin D (SMD - 0.30, 95\% CrIs - 0.49; - 0.11) and IA HA (SMD $-0.21,95 \%$ CrIs $-0.31 ;-0.11$ ).

Conclusion Six months of treatment with IA HA, pCGS, pCS, vitamin D and the combination of IA HA and triamcinolone improve pain and/or physical function in patients suffering from knee OA.
\end{abstract}

\section{Introduction}

Electronic supplementary material The online version of this article (https://doi.org/10.1007/s40265-020-01423-8) contains supplementary material, which is available to authorized users.

C. Beaudart

c.beaudart@uliege.be

1 WHO Collaborating Center for Public Health Aspects of Musculoskeletal Health and Ageing, Division of Public Health, Epidemiology and Health Economics, University of Liège, Avenue Hippocrate 13, CHU Sart Tilman, Bât B23, 4000 Liege, Belgium

2 Geriatrics Department, Rehabilitation Research Group, Hospital del Mar Medical Research Institute (IMIM), Universitat Pompeu Fabra, Barcelona, Spain

3 Chair for Biomarkers of Chronic Diseases, Biochemistry Department, College of Science, King Saud University, Riyadh, Saudi Arabia
Osteoarthritis (OA) is a highly prevalent degenerative agerelated disease characterized by joint pain and function disability leading to clinically relevant outcomes including loss of motion, impairment in quality of life, and a higher risk of mortality [1-3]. The incidence of OA is rising due to the increase in life expectancy and in the prevalence of obesity, and is responsible of an ever-growing societal burden $[4,5]$. The knee is the most common location of OA and leads to the highest clinical and financial burden $[6,7]$.

The objectives of treating OA are to reduce symptoms and presumably to decrease disease progression. Several pharmacological treatments aiming at a better symptomatic control of OA are currently prescribed, but their efficacy has been widely challenged. Discrepancies observed in the treatment guidelines recently issued by various respected scientific societies-for example, the European Society for Clinical and Economic Aspects of Osteoporosis, Osteoarthritis 


\section{Key Points}

Because the efficacy of pharmacological treatments aiming at a better symptomatic control of osteoarthritis is often disputed, we performed a systematic review and network meta-analysis to assess the efficacy on pain and function of the drugs which are most widely prescribed against knee osteoarthritis.

Network meta-analysis including 79 randomized controlled trials (15,609 individuals), showed that pain was improved following 6 months of treatment with intraarticular hyaluronic acid, prescription-grade crystalline glucosamine sulfate, prescription-grade chondroitin sulfate, vitamin D and the combination of IA hyaluronic acid and triamcinolone.

Network meta-analysis including 55 randomized controlled trials $(13,655$ individuals $)$ showed that function was improved following 6 months of treatment with intra-articular hyaluronic acid, prescription-grade crystalline glucosamine sulfate, and vitamin D.

and Musculoskeletal Diseases (ESCEO) [8-10], the Osteoarthritis Research Society International (OARSI) [11], the American College of Rheumatology (ACR) [12], the European League Against Rheumatism (EULAR) [13]—generate confusion and uncertainties in the minds of clinicians responsible for the daily management of knee OA.

Network-meta-analyses (NMA) are seen as providing a high level of scientific evidence. They allow comparison of different treatments, making direct and indirect comparisons possible within a network of randomized controlled trials. Such analyses enable measurement, in a robust and unbiased way, of the efficacy of an intervention in a clinical context. To date, one single NMA has been published to assess the efficacy of the symptomatic treatments used in knee OA [14]. Whereas methodologically robust, the conclusions of this NMA are difficult to translate into daily practice because of its time-horizon set up at 12 months. Even if OA is a progressive chronic disorder that requires long-term management, a very restricted number of treatments are prescribed continuously for 12 months or have a carry-over effect maintained for up to 1 year.

To assess and compare the efficacy of a practically relevant panel of knee OA treatments, we developed an NMA with a time-horizon of 6 months. Such a duration is consistent with the prescribing practices of OA clinicians. It is also the duration of treatment recommended by the European Medicines Agency (EMA) for studies of new chemical entities applying for a Marketing Authorization for the symptomatic treatment for OA [15]. We selected pain and function as the outcomes of our NMA to be consistent with the EMA and with the US Food and Drug Administration (FDA), which recommend them as being the two co-primary endpoints required for the assessment of symptomatic drugs in OA.

\section{Methods}

The proposed systematic review and network MA was conducted and reported in accordance with the Preferred Reporting Items for Systematic Review and Meta-analysis (PRISMA) using the Extension Statement for Reporting of Systematic Reviews Incorporating Network Meta-analyses of Health Care Interventions (PRISMA-NMA) [16] [the completed PRISMA-NMA is available in Supplementary Table A1, Electronic Supplementary Material (ESM)]. A protocol was developed and published in PROSPERO (CRD42020163194) prior to the conduct of the network meta-analysis.

Our research project can be summarized with the following PICOs format: P (Population): knee osteoarthritis; I (Intervention): any pharmacological treatment for knee OA administrated during a continuous period of 6 months or more (any form of treatment); C (Comparator): active control (another active pharmacological treatment), or placebo; O (Outcome): pain and function; S (Study design): randomized controlled trials (RCTs).

\subsection{Literature Search}

We searched MEDLINE (via Ovid), Scopus, and the Cochrane Database of Systematic Reviews (via Ovid) for RCTs assessing the efficacy of knee OA treatments published from inception of databases up to August 2019. The search was limited to English and French studies [17]. A combination of terms of Medical Subject Headings (MeSH) and keywords was used in search strategy (search strategies for each database available in the ESM, Table A2). Additionally, bibliographies of all included studies were manually checked for other potentially relevant publications. Moreover, references retrieved from previous network meta-analyses, meta-analyses, systematic reviews, and review articles performed on the same or a similar topic were hand searched and included if consistent with our selection criteria. We also searched on clinical trial registries (http://www.clini caltrial.gov) for potential unpublished studies and contacted experts in the field to obtain their opinions about our search strategy and the included papers.

The search results from the electronic sources and hand searching were imported in Covidence software for data management. 


\subsection{Study Selection}

Five researchers (CB, AG, LL, VL, and DSR) independently conducted the selection of references first based on title/ abstract with every single reference screened by two different reviewers. Any discrepancy was resolved through discussion and consensus meetings including all five researchers. The same process was used for data extraction and risk of bias assessment. As a second step, references were screened based on their full text. Excluded studies and reasons for exclusion were recorded for all studies excluded in the fulltext paper screening stage. Inclusion of studies was based on a list of inclusion/exclusion criteria reviewed by all the five researchers (Table 1).

\subsection{Data Extraction}

Data extraction was performed on an Excel sheet tested a priori on a sample of six RCTs to check for accuracy and consistency of the extraction file. The following data were extracted for each individual RCT: (1) General information related to the manuscript and population: first author, year of publication, country, characteristics of the population (sample size, groups, age, sex, body mass index, OA grade, and OA duration), funding source/conflicts of interest (COIs) of authors. (2) Information related to the treatment: type of pharmacological treatment, dose, route of administration, length of follow-up. (3) Information related to outcomes: type of outcome measured (pain, function), outcome measurement instrument/scale, results of the intervention (change from baseline to the end of follow-up period (6 months or more) or baseline data and end of follow-up results) for each outcome. For pain, when different scales were used within the same study for the measure of pain, we extracted, preferentially, first the results of the Western Ontario and McMaster Universities Arthritis Index (WOMAC) pain subscale, then the Visual Analogical Scale (VAS) (for pain during activity, pain during walking, global knee pain, pain at rest, pain during night), and then any other scales/subscales for measuring pain. For function, we extracted first the result of the WOMAC function subscale [18], and then any other scale/subscales measuring function. The Lequesne Index (LI) [19] was not considered as a scale of function and was therefore not included as outcome.

Authors of individual papers were contacted in case of any missing information.

\subsection{Risk of Bias Assessment}

The Cochrane Risk of Bias (RoB) tool was used to assess individual RCT quality [20]. Studies were assessed for sequence generation, allocation concealment, blinding of participants, study personnel and outcome assessors, incomplete outcome data and selective outcome reporting. With regard to blinding of participants and personnel, we considered explicit declarations by the authors that the study was conducted in a double-blind fashion to be sufficient for a low risk of bias judgment, provided that no contraindications were present. For studies with injectable products, where blinding of the person administering the product may not have been possible due to a difference in viscosity between the products, the study was considered to be at low risk of bias if the person carrying out the injection was not involved in any other aspect of the study and no other contraindications were present. For the item on incomplete outcome reporting, we only accorded a low risk of bias judgment to those studies that carried out their analyses on a (modified) intent-to-treat sample, either including all randomized subjects or all randomized subjects who took at least one dose of study product, or studies without any dropout.

\subsection{Data Synthesis}

All the analyses were performed using R package "netmeta" and extensions.

\subsubsection{Relative Treatment Effect}

Because studies can use different scales for measuring outcomes, changes from baseline to 6-month treatment or more (measured with WOMAC subscales, VAS scales, or various Likert scales) were translated into standardized mean difference (SMD, Cohen's $d$ ) effect size, defined as the difference in change from baseline between two interventions divided by the pooled standard difference (SD) of the difference.

For studies reporting different follow-up outcome results, the results of the longest follow-up period were extracted. Intent-to-treat data were used when available.

\subsubsection{Dealing with Missing Outcomes}

We extracted original mean differences and SDs, when available, in each individual study. When only baseline and follow-up values were available and not the mean differences and SDs, we estimated the mean differences and SDs from these baseline and follow-up values for each group individually using the formula proposed in the Cochrane Handbook for Systematic Reviews.

When standard errors (SEs) only were reported, we converted them to SDs. If neither SDs nor SEs were available, we estimated SDs from p-values or 95\% CrIs as described in the Cochrane Handbook for Systematic Reviews. If none of these options were manageable, we contacted the authors of individual studies to ask them for the missing information. Finally, our last strategies were to impute missing SDs from other studies using the same scale to measure the outcomes 
Table 1 Inclusion and exclusion criteria for study selection

\begin{tabular}{|c|c|}
\hline Inclusion criteria & Exclusion criteria \\
\hline \multicolumn{2}{|l|}{ Participants } \\
\hline \multicolumn{2}{|l|}{ Age: adults $>18$ years } \\
\hline \multicolumn{2}{|l|}{ Sex: both sexes } \\
\hline Settings: any & Settings: pre-post surgical intervention \\
\hline \multicolumn{2}{|l|}{ Ethnicity: any } \\
\hline \multicolumn{2}{|l|}{ Co-morbidities: any } \\
\hline Condition: knee OA & $\begin{array}{l}\text { Condition: concomitant knee OA and hip OA with no separate results } \\
\text { for knee }\end{array}$ \\
\hline \multicolumn{2}{|l|}{ Intervention } \\
\hline $\begin{array}{l}\text { Treatment: acetaminophen/paracetamol, vitamin E, vitamin } \mathrm{D} \text {, hyalu- } \\
\text { ronic acid, methylprednisolone, triamcinolone, celecoxib, diclofenac, } \\
\text { etofenamate, etoricoxib, licofelone, naproxen, chondroitin sulfate, } \\
\text { diacerein, glucosamine sulfate (see the complete list with complete } \\
\text { names of treatments in the search strategy) }\end{array}$ & Any treatment not currently used for the management of OA \\
\hline $\begin{array}{l}\text { Type of treatments: monotherapy or any recognized combination of } \\
\text { treatments }\end{array}$ & $\begin{array}{l}\text { Type of treatments: combination of pharmacological treatments with } \\
\text { another pharmacological treatment not in the list of inclusion or } \\
\text { with non-pharmacological treatments (e.g. physical activity, manual } \\
\text { therapy, physiotherapy, etc.) }\end{array}$ \\
\hline \multicolumn{2}{|l|}{ Route of administration: any } \\
\hline \multicolumn{2}{|l|}{$\begin{array}{l}\text { Length of treatment: } 6 \text { consecutive months or }+ \text { (for IA injections, at } \\
\text { least } 6 \text { months of follow-up) }\end{array}$} \\
\hline \multicolumn{2}{|l|}{ Comparator } \\
\hline \multicolumn{2}{|l|}{ Placebo } \\
\hline \multicolumn{2}{|l|}{ Active control (other OA treatment) } \\
\hline \multicolumn{2}{|l|}{ Rescue medication } \\
\hline \multicolumn{2}{|l|}{$\begin{array}{l}\text { Accepted } \\
\text { Allowed to continue an active treatment of OA if dose is stable through- } \\
\text { out the study OR }\end{array}$} \\
\hline \multicolumn{2}{|l|}{ Outcome (at least one measure of) } \\
\hline \multicolumn{2}{|l|}{ Pain (WOMAC, VAS, SF-36, etc.) } \\
\hline \multicolumn{2}{|l|}{ Function (WOMAC, SF-36, etc.) } \\
\hline \multicolumn{2}{|l|}{ Study design } \\
\hline RCTs (blind or not blind) & Quasi-randomized trials \\
\hline $\begin{array}{l}\text { Cross-over trials (if randomized) for the first part of the study (if at least } \\
6 \text { months of treatment in this first phase) }\end{array}$ & Case report \\
\hline Open-label trials (if randomized) & $\begin{array}{l}\text { Case series } \\
\text { Post hoc analyses }\end{array}$ \\
\hline Congress abstracts of RCTs & $\begin{array}{l}\text { Protocols of RCTs } \\
\text { Phase II or III clinical trials (not published) }\end{array}$ \\
\hline \multicolumn{2}{|l|}{ Other } \\
\hline English and French & \\
\hline
\end{tabular}

or to extract and estimate missing data from figures. When only median and interquartile ranges were available, we used the formula proposed by Hozo et al. [21] to convert them into means and SDs.
SMDs were calculated according to the Cochrane Collaboration methods. 


\subsubsection{Network Meta-Analyses}

Prior to running network meta-analyses (NMA), we assessed the transitivity assumption, which implies that studies comparing different sets of intervention are sufficiently similar to provide valid indirect inferences.

Then, we ran a Bayesian network meta-analysis to synthetize evidence for each outcome. We assumed a single heterogeneity parameter for each network. The available evidence is presented in a network diagram in which the breadth of each edge is proportional to the inverse of the variance of the summary effect of each direct treatment comparison.

We then estimated the probability for each intervention to be ranked as the most effective for a particular outcome improvement, given the relative effect sizes as estimated in NMA. As described in Salanti et al. [22], we obtained a hierarchy of the competing interventions using the surface under the cumulative ranking curve (SUCRA) and mean ranks. SUCRA values are expressed as a percentage, showing the relative probability of an intervention to be among the best options. The higher value represents the higher probability of being the best option.

We also performed a statistical evaluation of consistency, which consists of the agreement between direct and indirect evidence. We employed both local and global methods to evaluate consistency. Local methods identify pairwise comparisons or loops of evidence that might be important sources of inconsistency. We also evaluated consistency globally, in the entire network, by calculating the design-by-treatment interaction $\left(X^{2}\right.$ test and $I^{2}$ for inconsistency). In case of inconsistency, we tried to find an explanation by analyzing the studies and performing sensitivity analyses.

\subsubsection{Sensitivity Analyses (Exploring Heterogeneity)}

We ran a sensitivity analysis by removing studies for which a high risk of bias had been found in any of the RoB domains.

\subsubsection{Publication Bias}

We ran a comparison-adjusted funnel plot for pain and function outcome comparing all treatments against placebo to detect the presence of any dominant publication bias in NMA. Order of treatment was generated according to the number of patients included for each treatment.

\section{Results}

\subsection{Literature Search}

After removing duplicates between the different databases, 9,697 studies were screened for titles and abstracts. Among these references, 348 were assessed for eligibility by reviewing full texts. Finally, 80 individual RCTs were included in this network meta-analysis (Fig. 1). References of included studies are available in the ESM. Reasons for exclusion of all papers excluded during the full-text eligibility stage are recorded in ESM Table A3.

\subsection{Characteristics of Included Studies}

Characteristics of the 80 individual studies included in the NMA are available in ESM Table A4. Studies were published from 1988 to 2019 and included between 40 and 1583 participants with a total of 15,713 individuals. All but one included patients from both genders. More than half of the studies were performed on participants 60 years and older and the mean duration of treatment was 50 months. Most studies included a two-arm study design, but 14 studies included a three-arm design, four studies included a fourarm design, and one other study included a five-arm design. Table 2 summarizes the different treatments included in this NMA with distribution across pain and function outcomes. Risk of bias assessment and summary are available in ESM Fig. A1 and ESM Fig. A2.

\subsection{Efficacy of Knee Osteoarthritis (OA) Treatments on Pain}

Pain was investigated in 79 studies (15,609 individuals) using 26 different treatments that led to 101 pairwise comparisons. The network diagram including those 79 studies is shown in Fig. 2.

A significant association with decreased pain was found for the combination of intra-articular (IA) HA and triamcinolone (SMD - 0.49, 95\% CrI - 0.78; - 0.19), vitamin D (SMD - 0.31, 95\% CrI - 0.56; - 0.06), IA HA (SMD $-0.29,95 \% \mathrm{CrI}-0.40 ;-0.17$ ), pCGS (SMD $-0.29,95 \%$ $\mathrm{CrI}-0.58 ;-0.004)$, and pCS (SMD - 0.26, 95\% CrI - 0.44; -0.08). For pain, the combination IA HA + triamcinolone had the highest probability of being the most effective treatment to reduce pain (SUCRA value of 0.88) (see network forest plot in Fig. 3). A league table that presents results of the network meta-analyses and pairwise meta-analyses is available in ESM Fig. A3.

Heterogeneity was significant $\left(p<0.001, I^{2} 69 \%\right)$ in the model but inconsistency statistics revealed no inconsistency between direct and indirect evidence (between-design 
Fig. 1 PRISMA flowchart of study selection

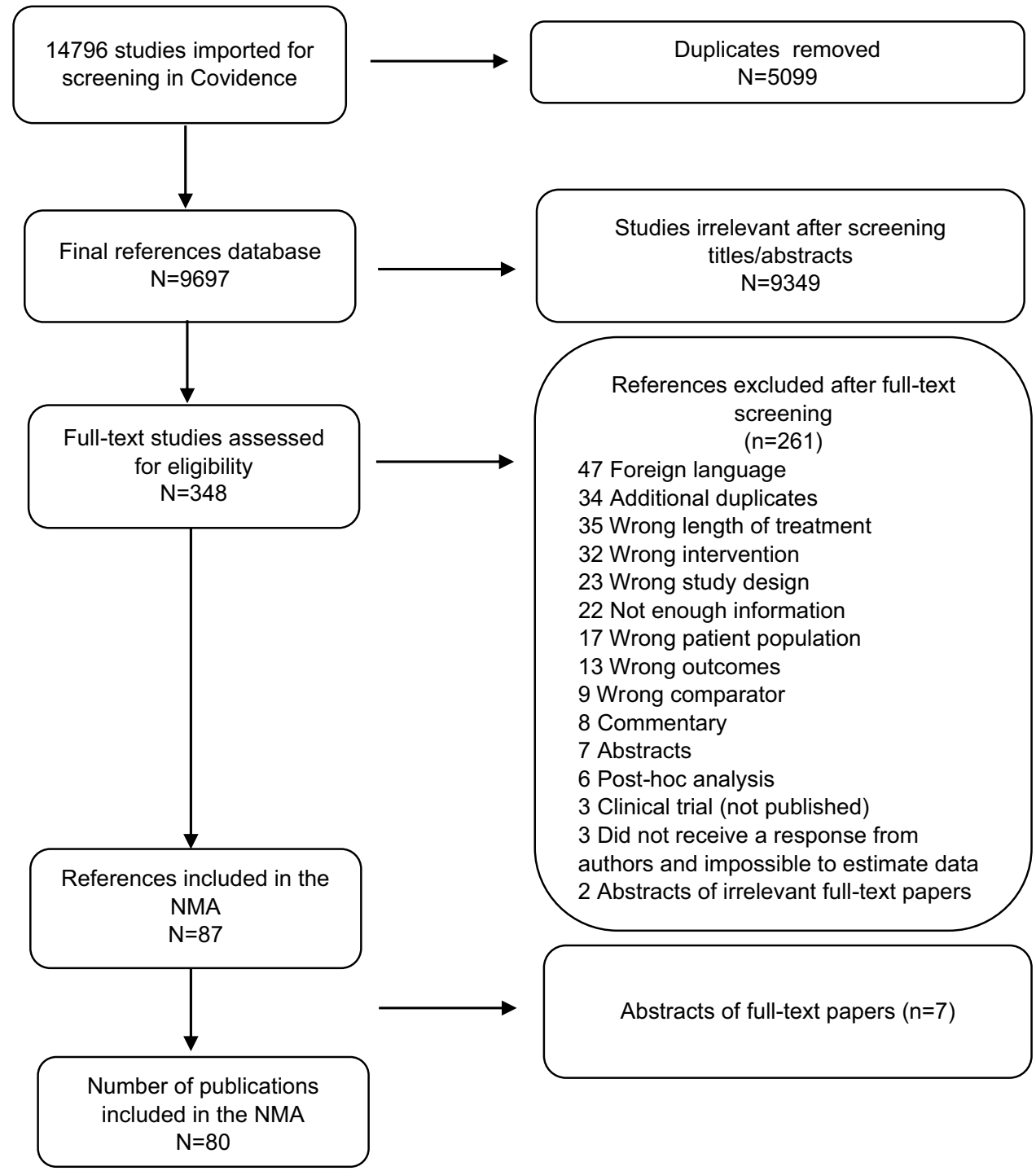

inconsistency $p=0.094)$. The comparison-adjusted funnel plot for pain efficacy of treatments against placebo suggested no funnel plot asymmetry (ESM Fig. A5).

In a sensitivity analysis, we included only studies with no risk of bias in any of the Cochrane RoB tool domains (i.e., we excluded studies with a high risk of bias in any domain), resulting in 30 studies being included (8754 individuals), 17 treatments and 43 pairwise comparisons. Results still demonstrated an effective effect on pain for pCGS, for the combination of IA HA + triamcinolone, for pCS for IA HA but also for IA methylprednisolone. With a SUCRA value of 0.79 , IA methylprednisolone was shown as having the highest probability of being the most effective treatment to reduce pain in knee OA in these sensitivity analyses. Within-design heterogeneity was still significant $(p<0.001)$ and between-design inconsistency was also significant $(p=0.023)$. A network forest plot of the sensitivity analyses is available in ESM Fig. A7.

\subsection{Efficacy of Knee OA Treatments on Function}

Function was investigated in 55 studies $(13,655$ individuals) using 21 different treatments that led to 84 pairwise comparisons. The following treatments were shown to increase function: pCGS (SMD - 0.44, 95\% CrI - 0.66; - 0.21), vitamin D (SMD - 0.30, 95\% CrI - 0.49; - 0.11) and IA HA (SMD $-0.21,95 \% \mathrm{CrI}-0.31 ;-0.11)$. pCGS had the highest probability to most improve function (SUCRA value of 0.91) (Fig. 4). A league table that presents results of the network meta-analyses and pairwise meta-analyses is available in ESM Fig. A4. On the contrary, piroxicam was shown to reduce function (SMD 0.83, 95\% CrI 0.02; 1.64).

Within-design heterogeneity was significant $\left(p<0.001, I^{2}\right.$ $52.1 \%)$ as well as between-design inconsistency $(p=0.024)$. The comparison-adjusted funnel plot for function efficacy of treatments against placebo suggested that there might be small-study effects in the NMA (ESM Fig. A6). 
Table 2 Number of studies that reported pain or function outcomes that were identified for each treatment

\begin{tabular}{|c|c|c|c|c|}
\hline \multirow[t]{2}{*}{ Treatment } & \multicolumn{2}{|l|}{ Pain } & \multicolumn{2}{|l|}{ Function } \\
\hline & Number of studies & Number of individuals & Number of studies & $\begin{array}{l}\text { Number of } \\
\text { individuals }\end{array}$ \\
\hline Placebo & 50 & 4570 & 38 & 4056 \\
\hline IA hyaluronic acid & 39 & 3049 & 25 & 2233 \\
\hline IA triamcinolone & 9 & 534 & 7 & 494 \\
\hline Prescription chondroitin sulfate & 10 & 1200 & 5 & 755 \\
\hline IA methypredinosolone & 7 & 586 & 2 & 245 \\
\hline Celecoxib & 6 & 1219 & 6 & 1214 \\
\hline Glucosamine sulfate & 5 & 548 & 5 & 726 \\
\hline Glucosamine + chondroitin sulfate & 5 & 787 & 5 & 914 \\
\hline IA hyaluronic acid + triamcinolone & 4 & 251 & 4 & 265 \\
\hline Naproxen & 4 & 636 & 2 & 446 \\
\hline Vitamin D & 4 & 498 & 4 & 571 \\
\hline Prescription crystalline glucosamine sulfate & 3 & 313 & 3 & 313 \\
\hline Diclofenac & 3 & 91 & 2 & 75 \\
\hline Vitamin E & 3 & 138 & 2 & 105 \\
\hline Acetaminophen/paracetamol & 2 & 181 & 1 & 108 \\
\hline Avocado soybean unsaponifiables & 2 & 236 & 2 & 236 \\
\hline Chondroitin sulfate & 2 & 370 & 4 & 631 \\
\hline Piroxicam & 1 & 51 & 1 & 52 \\
\hline IA condrotine & 1 & 36 & 1 & 36 \\
\hline IA prednisolone & 1 & 40 & 0 & 0 \\
\hline IA cortivazol & 1 & 25 & 0 & 0 \\
\hline IA dexamethasone & 1 & 25 & 0 & 0 \\
\hline IA etofenamate & 1 & 29 & 0 & 0 \\
\hline Etoricoxib & 1 & 33 & 1 & 33 \\
\hline IA ketorolac & 1 & 16 & 0 & 0 \\
\hline Licofelone & 1 & 147 & 1 & 147 \\
\hline
\end{tabular}

IA intra-articular

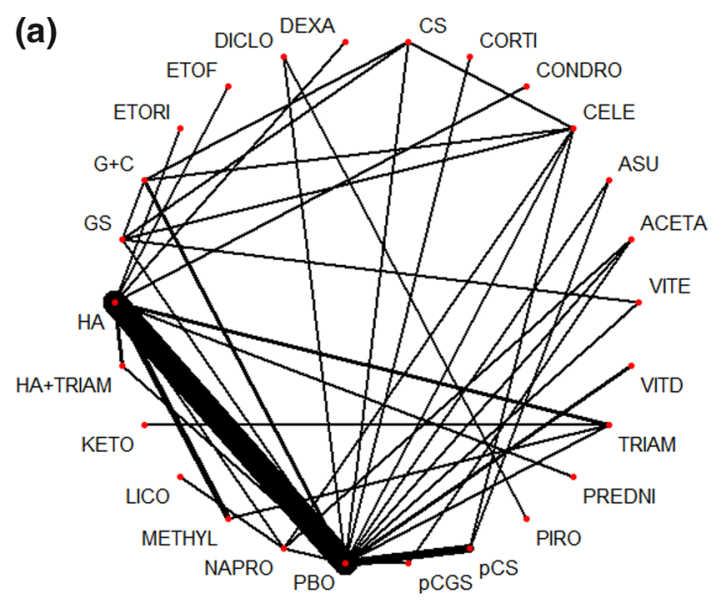

Fig. 2 Network plot of pain (a) and function (b) efficacy of knee osteoarthritis (OA) treatments (breadth of each edge is proportional to the inverse of the variance of the summary effect of each direct treatment comparison). ACETA acetaminophen/paracetamol, $A S U$ avocado soybean unsaponifiables, CELE celecoxib, CONDRO condrotide, CORTI cortivazol, $C S$ chondroitin sulfate, DEXA dexamethasone, DICLO diclofenac, ETOF etofenamate, ETORI etoricoxib, GS glucosamine

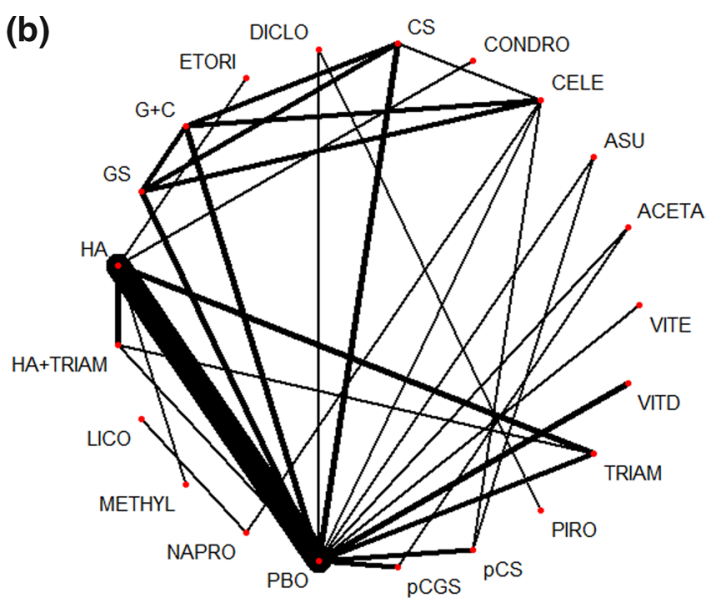

sulfate, $G+C$ combination of glucosamine sulfate + chondroitin sulfate, $H A$ hyaluronic acid, KETO ketorolac, LICO licofelone, METHYL methylprednisolone, NAPRO naproxen, $P B O$ placebo, $p C G S$ prescription-grade crystalline glucosamine sulfate, $p C S$ prescription grade chondroitin sulfate, PRED prednisolone, PIRO piroxicam, TRIAM triamcinolone, VITD vitamin D, VITE vitamin $\mathrm{E}$ 


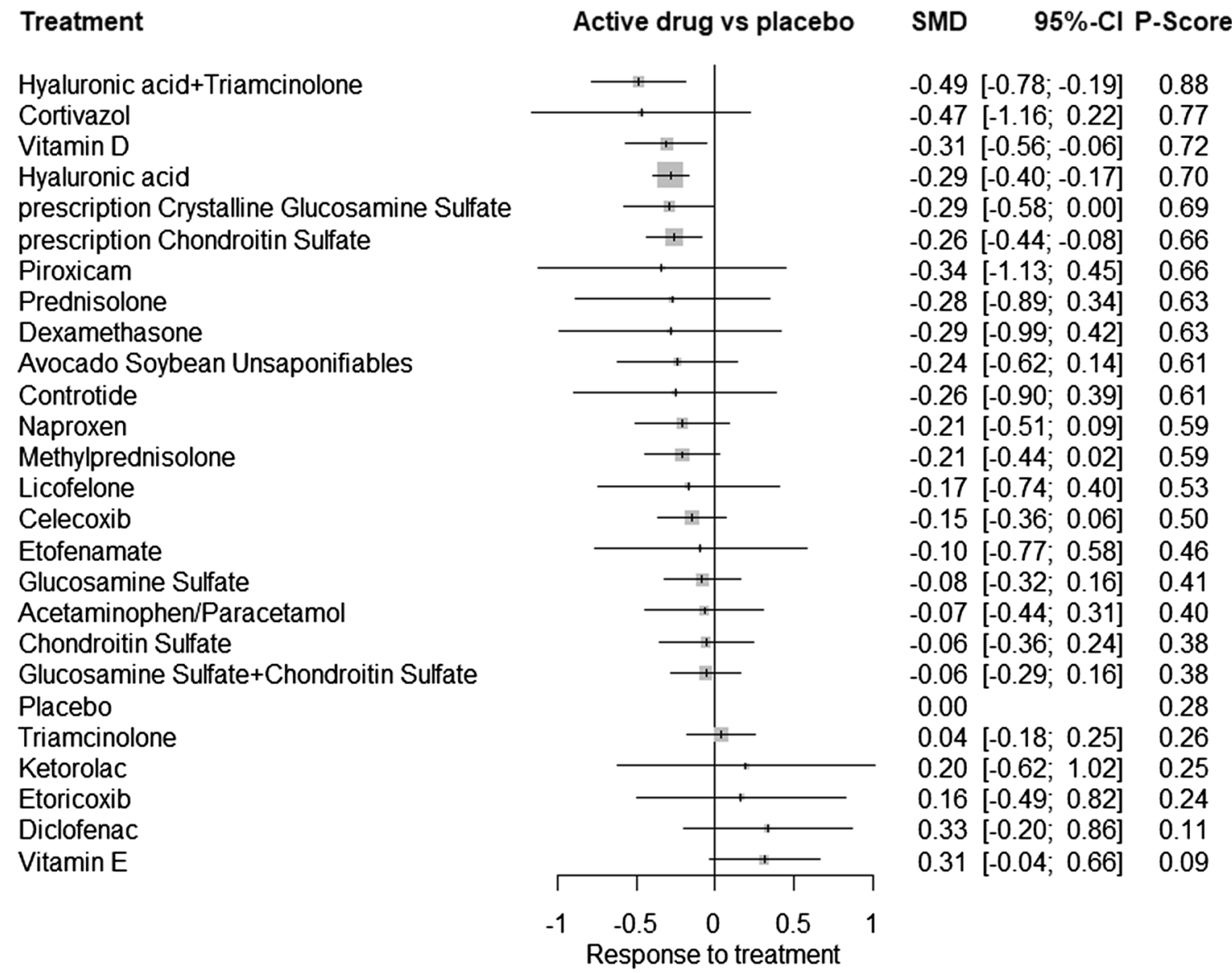

Fig. 3 Network meta-analysis forest plot summarizing the efficacity of knee osteoarthritis treatments in reducing pain

The sensitivity analysis, including only studies with no risk of bias in any of the Cochrane RoB tool domains, resulted in the inclusion of 22 studies (7566 individuals), 16 treatments and 33 pairwise comparisons. Results demonstrated a significant effect of pCGS (SUCRA value of 0.94, as having the highest probability of being the best treatment for increasing function) and vitamin $\mathrm{D}$ as effective on function in knee OA patients. Within-design heterogeneity was still significant $(p<0.001)$ but between-design inconsistency was not significant anymore $(p=0.2345)$. A network forest plot of the sensitivity analyses is available in ESM Fig. A8.

\subsection{Additional Analyses}

Effects of treatment on stiffness and joint space narrowing (JSN) have also been explored as additional analyses. Forest plots are presented in the ESM files (Fig. A9 and Fig. A10). A network meta-analysis on stiffness (38 RCTs, 10,049 individuals, 19 treatments, 49 pairwise comparisons) indicated that only chondroitin sulfate is effective against stiffness in knee osteoarthritis (SMD - 0.4 (95\%
CI $-0.74 ;-0.05)$. A network meta-analysis on JSN (14 RCTs, 3750 individuals, 12 treatment, 28 pairwise comparisons) concluded that both pCGS and pCS are effective to reduce JSN in knee osteoarthritis with a mean difference of JSN of 0.27 ( $95 \%$ CI 0.09 ; 0.46) mm for pCGS versus placebo and 0.13 (95\% CI $0.01 ; 0.25) \mathrm{mm}$ for $\mathrm{pCS}$ versus placebo, respectively.

\section{Discussion}

This network meta-analysis, based on 80 RCTs, assessed the efficacy of different chemical entities currently used for the symptomatic treatment of knee OA. Our results indicate that, when administered for 6 months or more, IA HA, pCGS, pCS, vitamin D, and the combination of IA HA + triamcinolone significantly improved pain and/or physical function in patients suffering from knee OA. Injections of $\mathrm{HA}+$ triamcinolone for pain, and pCGS for function, have respectively the highest probability of being the most effective treatment. 
Treatment

prescription Crystalline Glucosamine Sulfate
Diclofenac
Acetaminophen/Paracetamol
Vitamin D
Hyaluronic acid
Hyaluronic acid+Triamcinolone
Licofelone
prescription Chondroitin Sulfate
Avocado Soybean Unsaponifiables
Naproxen
Celecoxib
Condrotide
Chondroitin Sulfate
Glucosamine Sulfate
Glucosamine Sulfate+Chondroitin Sulfate
Triamcinolone
Placebo
Methylprednisolone
Vitamin E
Etoricoxib
Piroxicam

Active drug vs placebo

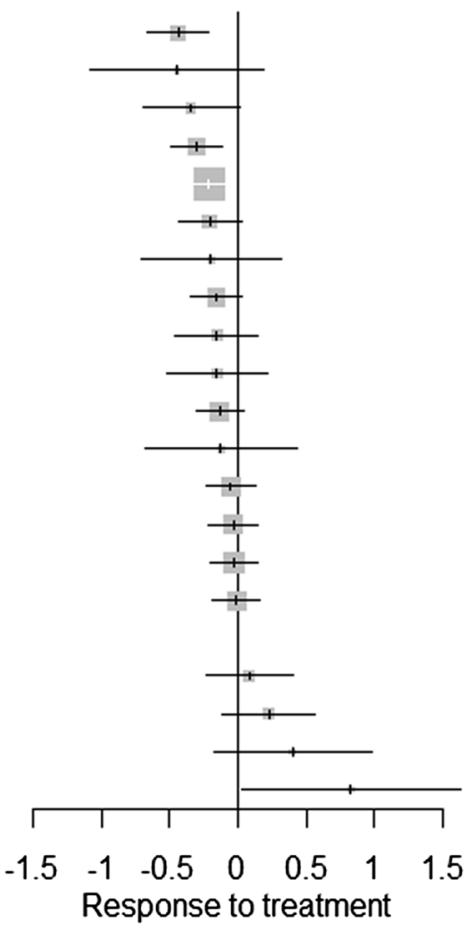

SMD

95\%-Cl P-Score

$\begin{array}{ll}-0.44[-0.66 ;-0.21] & 0.91 \\ -0.45[-1.08 ; 0.19] & 0.82 \\ -0.34[-0.70 ; 0.01] & 0.80 \\ -0.30[-0.49 ;-0.11] & 0.80 \\ -0.21[-0.31 ;-0.11] & 0.69 \\ -0.21[-0.44 ; 0.03] & 0.67 \\ -0.19[-0.71 ; 0.32] & 0.62 \\ -0.16[-0.35 ; 0.03] & 0.60 \\ -0.15[-0.46 ; 0.15] & 0.58 \\ -0.15[-0.52 ; 0.22] & 0.57 \\ -0.13[-0.31 ; 0.05] & 0.56 \\ -0.12[-0.67 ; 0.43] & 0.53 \\ -0.05[-0.23 ; 0.13] & 0.41 \\ -0.03[-0.21 ; 0.14] & 0.38 \\ -0.03[-0.20 ; 0.14] & 0.37 \\ -0.01[-0.19 ; 0.16] & 0.35 \\ 0.00 & \\ 0.09[-0.23 ; 0.40] & 0.31 \\ 0.23[-0.11 ; 0.57] & 0.25 \\ 0.40[-0.18 ; 0.98] & 0.11 \\ 0.83[0.02 ; 1.64] & 0.03\end{array}$

0.91

0.82

.80

.80

69

60

.58

0.57

56

.41

\subsection{7}

0.35 .31 0.25 0.11 0.03

Fig. 4 Network meta-analysis forest plot summarizing the efficacity of knee osteoarthritis treatments in improving function

So far, only one NMA [14] previously investigated the effects of pharmacological treatments on pain and function in patients with knee OA. In this elegant publication, the authors selected a time-horizon of 12 months instead of the 6 months that we preferred for our present study. They included 31 RCTs for pain and 13 for physical function, and found significant improvement in pain and function with pCGS only. The main variable explaining why we identified more treatments potentially affecting knee OA symptoms is the time horizon. Indeed, we included studies of a 6-month duration rather than concentrating only on duration of treatments of 12 months or more. We subsequently were able to include a larger number of RCTs. In fact, several treatments, such as non-steroidal anti-inflammatory drugs (NSAIDs) or analgesics, are generally prescribed for a shorter duration than 12 months, because of safety concerns [8, 9, 23]. Therefore, clinical trials assessing the efficacy of these medications were often limited to shorter periods rather than exposing patients for a 12-month continuous administration. Moreover, some IA treatments, such as HA, are commonly administered in sequences including one to five injections, with intervals between doses usually not exceeding 3 weeks. Albeit these treatments are known to generate a prolonged benefit, the persistence of their symptomatic effect is seldom still persistent 12 months after the first injection, which explains why studies assessing these medications are often limited to 6 months [8].

Oral NSAIDS are widely prescribed in OA and their use is recommended by most recently published clinical guidelines [8, 11-13]. However, we were unable to demonstrate a statistically significant benefit of any NSAID on pain or function, after 6 months, in knee OA patients. It is noteworthy that both celecoxib and naproxen showed trends towards a beneficial effect on OA symptoms but the numerical improvement observed with these two NSAIDs did not reach the level of statistical significance in our study. Once again, this may reflect the fact that oral NSAIDs are mainly recommended for intermittent use or short-term courses, rather than prolonged continuous treatments $[8,11]$. A recent systematic review and meta-analysis (MA) concluded that the NSAID-induced symptomatic benefit observed in knee OA peaks after 2 weeks while the drug-related cardiovascular and gastrointestinal adverse events reach statistical significance as early as 4 weeks [24]. The optimal time-horizon of a MA or NMA aiming at a specific assessment of the symptomatic effect of NSAIDs in OA should probably be much shorter than 6 months. Surprisingly, we also observed a negative effect of piroxicam on function as compared to placebo. Looking closer to the results, only one study, by La Montagna et al. [25], investigated effects of piroxicam on function, and the comparator used in this study was 
diclofenac. The results provided by the network analysis should therefore be interpreted with caution given that only one study is included and the observed effect against placebo is the result of indirect analyses.

Symptomatic slow-acting drugs for OA (SYSADOAs) are recommended by some but not by all guidelines as a useful addition to the armamentarium against OA [8, 11-13]. It has been suggested that discrepancies between the therapeutic value given to glucosamine or chondroitin products in the various guidelines may be related to the fact that some of the guidance documents distinguish pharmaceutical-grade or prescription-only preparations from generics, nutraceuticalgrade or over-the-counter (OTC) formulations, while other guidelines consider these products as a whole class with no attempt to separate them by grade or brand $[26,27]$. Since a Cochrane Review and two more recent MAs, conducted independently from any corporate funding, concluded that all GS or CS products do not share the same level of symptomatic efficacy in OA [28-30], our NMA considered separately the prescription-grade chondroitin sulfate (pCS) and the prescription-grade crystalline glucosamine sulfate (pCGS) from the other chondroitin or glucosamine products tested in OA studies. Our results support the view that pCS and pCGS are more effective in reducing pain in knee OA than other glucosamine or chondroitin preparations, which do not provide any significant clinical benefit, as outlined in previous publications $[8,11,12]$. This difference between the various glucosamine and chondroitin preparations was confirmed in our sensitivity analysis excluding studies with a high risk of bias.

The effect of pCS on function was only borderline significant (SMD - 0.16; 95\% CI -0.3; 0.04). Several studies of pCS in knee OA selected the Lequesne index (LI) as the assessment tool for function. Although a validated instrument for measuring the algo-functional impact of $\mathrm{OA}, \mathrm{LI}$ is a composite index that does not allow discriminating between the respective components of pain and function, as the different subscales of the WOMAC do [18, 19]. Since we felt it important to separately identify the effects of the different drugs on pain and function, we discarded from the analysis trials that did not provide separate results of the investigational product on these two symptoms of OA. We cannot exclude that if a specific tool assessing function apart from pain would have been selected in the pCS trials, this might have positively influenced the outcomes of our assessment of the effect of pCS on function.

Acetaminophen/paracetamol is not associated with a significant clinical benefit in knee OA. Our results support previous reports suggesting that the magnitude of the effect of this compound in $\mathrm{OA}$ is at best trivial and not clinically relevant in patients with knee or hip OA [31, 32]. This observation, combined with increasing evidence that acetaminophen/paracetamol is linked to a considerable level of toxicity, particularly at the upper end of standard analgesic doses [33, 34], translated into a negative recommendation for the prolonged use of this medication in knee OA in all the recently published guidelines $[8,11,12]$.

A large number of RCTs investigated the effects of IA injections of HA and/or glucocorticosteroids (GCST) on pain and function in knee OA. The combination of IA HA and triamcinolone, a well-known GCST, was ranked as the most effective approach to reduce pain in the general NMA and in the sensitivity analysis. Injection of HA alone was also beneficial at a 6-month horizon, both on pain and on function, while IA administration of GCST alone did not provide a similar benefit. This is perfectly in accordance with the established pharmacodynamic properties of the two products: IA GCST produces a fast but transient benefit, which can hardly be expected to last up to 6 months, whereas IA HA needs some more latency to show its maximal effect, which is expected to be long-lasting for several weeks or months [8, 35-37].

Vitamin D reduced pain and improved function in knee OA patients. While deficiency in endogenous vitamin $\mathrm{D}$, reflected by low levels of circulating $25 \mathrm{OH}$ vitamin $\mathrm{D}$, was associated with the onset and progression of knee OA [38], maybe through an increased frequency of medial meniscus lesions [39], results of individual RCTs were inconclusive [40-43]. Their outcomes varied from a non-significant trend for symptom reduction [40-42] to a small but statistically significant benefit on pain and function after 12 months of administration [43].

Our decision to limit this NMA to treatments that are currently prescribed or recommended in daily practice explains why we did not assess treatments that are currently under investigation (e.g., tanezumab, fanizumab, lorecicivint, etc.) or which, despite their availability for other indications, are not or no longer commonly prescribed against OA (e.g., strontium ranelate, bisphosphonates, calcitonin, doxycycline, etc.). We did not include non-pharmacological interventions in this NMA neither, albeit all evidence-based guidelines agree that the medical management of OA should include both non-pharmacological and pharmacological treatment modalities [44]. The main differences observed between the recently published recommendations $[8,11,12]$ and the subsequent confusion that these discrepant opinions introduce in the mind of the prescribers are linked to the respective value given to the different available medications. We thus tried to concentrate our efforts on a fair and unbiased assessment of the respective symptomatic benefit that can be expected from these medications after a 6-month treatment.

We considered stiffness as an ancillary outcome of our MNA. Indeed, stiffness is not recommended as an appropriate endpoint in the EMA or FDA guidelines for the assessment of new chemical entities aiming at the symptomatic 
management of knee OA. We also observed a large heterogeneity across studies in the tools that were used to report this outcome, making its assessment poorly reliable. Our results reporting a reduction in JSN with pCGS and with pCS are in perfect accordance with previous publications showing that pCGS $[45,46]$ or pCS [47] are able to significantly prevent structural progression of osteoarthritis. However, in the context of the present investigation, these analyses should be interpreted with caution because the time-horizon selected for our NMA was 6 months. Magnetic resonance imaging (MRI) may provide rapid information on the progression of knee OA, hence presumably allowing assessment of the structure-modifying properties of a drug after a couple of months only [48]. However, until now, JSN measured on plain X-rays has been most often used as the primary outcome of studies investigating a possible effect of a medication on OA structural progression [49]. All studies that assessed the impact of a drug on JSN had a much longer duration than 6 months, often up to 2 years $[45,50]$. Therefore, assessing the structuremodifying properties of the treatments included in this MNA within such a short timeframe (6 months) could be seen as disputable.

Finally, we also discovered a potential small study effect in the funnel plot on function. Several potential explanations may be publication bias, selective reporting outcomes, or even clinical heterogeneity between patients included in large versus in small studies. Most of these factors are nevertheless out of our control. To restrict a maximum publication bias issue, we performed comprehensive and systematic research of the literature. The only limitations of our search strategy were that we decided to restrict our search to French and English studies, which may have introduced publication bias but also that our search was limited to August 2019 . This last point is a classical issue in conducting and publishing meta-analyses, which is a lengthy process. However, we truly believed that, due to our inclusion criteria, we would have missed a very restricted number of new studies, with a very low probability of significantly altering the current results.

\section{Conclusion}

In this systematic review and network meta-analysis that included 80 RCTs, we highlighted that 6 months of treatment of knee OA patients with intra-articular hyaluronic acid, prescription-grade crystalline glucosamine sulfate, prescription-grade chondroitin sulfate, vitamin $\mathrm{D}$, and the combination of intra-articular hyaluronic acid and triamcinolone are effective to improve pain and/or function.

\section{Declarations}

Funding None.

Conflict of interest CB, LL, VL, AG, DSR: None. OB: Grants or fees: Amgen, Aptissen, Biophytis, IBSA, MEDA, Sanofi, Servier, SMB, Theramex and UCB. JYR: Consulting fees or paid advisory boards: IBSA-GENEVRIER, MYLAN, RADIUS HEALTH, PIERRE FABRE, ECHOLIGHT, TEVA. Lecture fees when speaking at the invitation of sponsor: IBSA-GENEVRIER, MYLAN, CNIEL, DAIRY RESEARCH COUNCIL (DRC), TEVA. Grant Support from Industry (All through Institution): IBSA-GENEVRIER, MYLAN, CNIEL, RADIUS HEALTH.

Availability of data and materials Under request.

Code availability Under request.

Author contributions $\mathrm{CB}$, JYR, and $\mathrm{OB}$ conceived and designed the study. CB, AG, VL, LL, and DSR selected the articles and extracted the data. CB and LL analyzed the data. CB wrote the first draft of the manuscript. All authors were involved in the interpretation of data and contribution to the writing of the final manuscript.

Open Access This article is licensed under a Creative Commons Attribution-NonCommercial 4.0 International License, which permits any non-commercial use, sharing, adaptation, distribution and reproduction in any medium or format, as long as you give appropriate credit to the original author(s) and the source, provide a link to the Creative Commons licence, and indicate if changes were made. The images or other third party material in this article are included in the article's Creative Commons licence, unless indicated otherwise in a credit line to the material. If material is not included in the article's Creative Commons licence and your intended use is not permitted by statutory regulation or exceeds the permitted use, you will need to obtain permission directly from the copyright holder. To view a copy of this licence, visit http://creativecommons.org/licenses/by-nc/4.0/.

\section{References}

1. Kanis JA, Cooper C, Rizzoli R, Reginster J-YY, Scientific Advisory Board of the European Society for Clinical and Economic Aspects of Osteoporosis (ESCEO) and the Committees of Scientific Advisors and National Societies of the International Osteoporosis Foundation (IOF). European guidance for the diagnosis and management of osteoporosis in postmenopausal women. Osteoporos Int. 2019;30:235-8.

2. Murray CJL, Vos T, Lozano R, Naghavi M, Flaxman AD, Michaud C, et al. Disability-adjusted life years (DALYs) for 291 diseases and injuries in 21 regions, 1990-2010: a systematic analysis for the Global Burden of Disease Study 2010. Lancet. 2012;380:2197-223.

3. Beaudart C, Biver E, Bruyère $\mathrm{O}$, Cooper $\mathrm{C}, \mathrm{Al}$-Daghri N, Reginster J-Y, et al. Quality of life assessment in musculo-skeletal health. Aging Clin Exp Res. 2018;30(5):413-8.

4. Litwic A, Edwards M, Dennison E, Cooper C. Epidemiology and burden of osteoarthritis. Br Med Bull. 2013;105:185.

5. Safiri S, Kolahi AA, Hoy D, Smith E, Bettampadi D, Mansournia MA, et al. Global, regional and national burden of rheumatoid arthritis 1990-2017: a systematic analysis of the Global Burden of Disease study 2017. Ann Rheum Dis. 2019;78:1463-71. 
6. Vos T, Allen C, Arora M, Barber RM, Bhutta ZA, Brown A, et al. Global, regional, and national incidence, prevalence, and years lived with disability for 310 diseases and injuries, 1990-2015: a systematic analysis for the Global Burden of Disease Study 2015. Lancet. 2016;388:1545-602.

7. Hiligsmann M, Cooper C, Arden N, Boers M, Branco JC, Luisa Brandi M, et al. Health economics in the field of osteoarthritis: an expert's consensus paper from the European Society for Clinical and Economic Aspects of Osteoporosis and Osteoarthritis (ESCEO). Semin Arthritis Rheum. 2013;43:303-13.

8. Bruyère $\mathrm{O}$, Honvo $\mathrm{G}$, Veronese $\mathrm{N}$, Arden NK, Branco J, Curtis EM, et al. An updated algorithm recommendation for the management of knee osteoarthritis from the European Society for Clinical and Economic Aspects of Osteoporosis, Osteoarthritis and Musculoskeletal Diseases (ESCEO). Semin Arthritis Rheum. 2019;49:337-50.

9. Bruyère $\mathrm{O}$, Cooper C, Pelletier J-PP, Branco J, Luisa Brandi M, Guillemin F, et al. An algorithm recommendation for the management of knee osteoarthritis in Europe and internationally: a report from a task force of the European Society for Clinical and Economic Aspects of Osteoporosis and Osteoarthritis (ESCEO). Semin Arthritis Rheum. 2014;44:253-63.

10. Kanis JA, Cooper C, Rizzoli R, Reginster JY. Executive summary of European guidance for the diagnosis and management of osteoporosis in postmenopausal women. Aging Clin Exp Res. 2019;31:15-7.

11. Bannuru RR, Osani MC, Vaysbrot EE, Arden NK, Bennell K, Bierma-Zeinstra SMA, et al. OARSI guidelines for the non-surgical management of knee, hip, and polyarticular osteoarthritis. Osteoarthr Cartil. 2019;27:1578-89.

12. Kolasinski SL, Neogi T, Hochberg MC, Oatis C, Guyatt G, Block J, et al. 2019 American College of Rheumatology/Arthritis Foundation Guideline for the management of osteoarthritis of the hand, hip, and knee. Arthritis Rheumatol. 2020;72:220-33.

13. Jordan KM. EULAR Recommendations 2003: an evidence based approach to the management of knee osteoarthritis: report of a Task Force of the Standing Committee for International Clinical Studies Including Therapeutic Trials (ESCISIT). Ann Rheum Dis. 2003;62:1145-55.

14. Gregori D, Giacovelli G, Minto C, Barbetta B, Gualtieri F, Azzolina $\mathrm{D}$, et al. Association of pharmacological treatments with long-term pain control in patients with knee osteoarthritis: a systematic review and meta-analysis. JAMA. 2018;320(24):2564-79.

15. European Medicines Agency. Guideline on clinical investigation of medicinal products used in the treatment of osteoarthritis. Available from http://www.ema.europa.eu. Accessed Aug 2020.

16. Hutton B, Salanti G, Caldwell DM, Chaimani A, Schmid CH, Cameron $\mathrm{C}$, et al. The PRISMA extension statement for reporting of systematic reviews incorporating network meta-analyses of health care interventions: checklist and explanations. Ann Intern Med. 2015;162:777.

17. Morrison A, Polisena J, Husereau D, Moulton K, Clark M, Fiander $\mathrm{M}$, et al. The effect of English-language restriction on systematic review-based meta-analyses: a systematic review of empirical studies. Int J Technol Assess Health Care. 2012;28:138-44.

18. Bellamy N, Buchanan WW, Goldsmith $\mathrm{CH}$, Campbell J, Stitt LW. Validation study of WOMAC: a health status instrument for measuring clinically important patient relevant outcomes to antirheumatic drug therapy in patients with osteoarthritis of the hip or knee. J Rheumatol. 1988;15:1833-40.

19. Lequesne MG, Mery C, Samson M, Gerard P. Indexes of severity for osteoarthritis of the hip and knee: validation-value in comparison with other assessment tests. Scand J Rheumatol. 1987;16:85-9.
20. Higgins JPT, Altman DG, Gøtzsche PC, Jüni P, Moher D, Oxman $\mathrm{AD}$, et al. The Cochrane Collaboration's tool for assessing risk of bias in randomised trials. BMJ. 2011;343:d5928.

21. Hozo SP, Djulbegovic B, Hozo I. Estimating the mean and variance from the median, range, and the size of a sample. BMC Med Res Methodol. 2005;5:13.

22. Salanti G, Ades AE, Ioannidis JP. Graphical methods and numerical summaries for presenting results from multiple-treatment meta-analysis: an overview and tutorial. J Clin Epidemiol. 2011;64:163-71.

23. Honvo G, Leclercq V, Geerinck A, Thomas T, Veronese N, Charles A, et al. Safety of topical non-steroidal anti-inflammatory drugs in osteoarthritis: outcomes of a systematic review and metaanalysis. Drugs Aging. 2019;36:45-64.

24. Osani MC, Vaysbrot EE, Zhou M, McAlindon TE, Bannuru RR. Duration of symptom relief and early trajectory of adverse events for oral nonsteroidal antiinflammatory drugs in knee osteoarthritis: a systematic review and meta-analysis. Arthritis Care Res. 2020;72(5):641-51

25. La Montagna G, Tirri G, Cacace E, Perpignano G, Covelli M, Pipitone V, et al. Quality of life assessment during six months of NSAID treatment [Gonarthrosis and Quality of Life (GOAL) Study]. Clin Exp Rheumatol. 1998;16:49-54.

26. Bruyère O, Cooper C, Al-Daghri NM, Dennison EM, Rizzoli R, Reginster J-Y. Inappropriate claims from non-equivalent medications in osteoarthritis: a position paper endorsed by the European Society for Clinical and Economic Aspects of Osteoporosis, Osteoarthritis and Musculoskeletal Diseases (ESCEO). Aging Clin Exp Res. 2018;30:111-7.

27. Honvo G, Bruyere O, Reginster J-Y. Update on the role of pharmaceutical-grade chondroitin sulfate in the symptomatic management of knee osteoarthritis. Aging Clin Exp Res. 2019;31:1163-7.

28. Honvo G, Bruyère O, Geerinck A, Veronese N, Reginster JY. Efficacy of chondroitin sulfate in patients with knee osteoarthritis: a comprehensive meta-analysis exploring inconsistencies in randomized, placebo-controlled trials. Adv Ther. 2019;36(5):1085-99.

29. Towheed T, Maxwell L, Anastassiades TP, Shea B, Houpt JB, Welch V, et al. Glucosamine therapy for treating osteoarthritis. Cochrane Database Syst Rev 2005;(2):CD002946.

30. Eriksen P, Bartels EM, Altman RD, Bliddal H, Juhl C, Christensen R. Risk of bias and brand explain the observed inconsistency in trials on glucosamine for symptomatic relief of osteoarthritis: a meta-analysis of placebo-controlled trials. Arthritis Care Res. 2014;66:1844-55.

31. da Costa BR, Reichenbach S, Keller N, Nartey L, Wandel S, Jüni $\mathrm{P}$, et al. Effectiveness of non-steroidal anti-inflammatory drugs for the treatment of pain in knee and hip osteoarthritis: a network meta-analysis. Lancet. 2017;390:e21-33.

32. Machado GC, Maher CG, Ferreira PH, Pinheiro MB, Lin CWC, Day RO, et al. Efficacy and safety of paracetamol for spinal pain and osteoarthritis: systematic review and meta-analysis of randomised placebo controlled trials. BMJ. 2015;350:h1225.

33. Roberts E, Nunes VD, Buckner S, Latchem S, Constanti M, Miller P, et al. Paracetamol: not as safe as we thought? A systematic literature review of observational studies. Ann Rheum Dis. 2016;75:552-9.

34. Conaghan PG, Arden N, Avouac B, Migliore A, Rizzoli R. Safety of paracetamol in osteoarthritis: what does the literature say? Drugs Aging. 2019;36:7-14.

35. Maheu E, Rannou F, Reginster JY. Efficacy and safety of hyaluronic acid in the management of osteoarthritis: evidence from real-life setting trials and surveys. Semin Arthritis Rheum. 2016;45:S28-33.

36. Honvo G, Reginster JY, Rannou F, Rygaert X, Geerinck A, Rabenda V, et al. Safety of intra-articular hyaluronic acid 
injections in osteoarthritis: outcomes of a systematic review and meta-analysis. Drugs Aging. 2019;36:101-27.

37. Cooper $\mathrm{C}$, Rannou F, Richette $\mathrm{P}$, Bruyère $\mathrm{O}, \mathrm{Al}-\mathrm{Daghri} \mathrm{N}$, Altman $\mathrm{RD}$, et al. Use of intraarticular hyaluronic acid in the management of knee osteoarthritis in clinical practice. Arthritis Care Res. 2017;69:1287-96.

38. Jin X, Antony B, Wang X, Persson MS, McAlindon T, Arden NK, et al. Effect of vitamin D supplementation on pain and physical function in patients with knee osteoarthritis (OA): an OA Trial Bank protocol for a systematic review and individual patient data (IPD) meta-analysis. BMJ Open. 2020;10:e035302.

39. Bassiouni H, Aly H, Zaky K, Abaza N, Bardin T. Probing the relation between vitamin $\mathrm{D}$ deficiency and progression of medial femoro-tibial osteoarthitis of the knee. Curr Rheumatol Rev. 2016;13:65-71.

40. Arden NK, Cro S, Sheard S, Dore CJ, Bara A, Tebbs SA, et al. The effect of vitamin D supplementation on knee osteoarthritis, the VIDEO study: a randomised controlled trial. Osteoarthr Cartil. 2016;24:1858-66.

41. Jin X, Jones G, Cicuttini F, Wluka A, Zhu Z, Han W, et al. Effect of vitamin D supplementation on tibial cartilage volume and knee pain among patients with symptomatic knee osteoarthritis: a randomized clinical trial. JAMA. 2016;315:1005-13.

42. McAlindon T, LaValley M, Schneider E, Nuite M, Lee JY, Price LL, et al. Effect of vitamin D supplementation on progression of knee pain and cartilage volume loss in patients with symptomatic osteoarthritis: a randomized controlled trial. JAMA. 2013;309:155-62.

43. Sanghi D, Mishra A, Sharma AC, Singh A, Natu SM, Agarwal S, et al. Does vitamin D improve osteoarthritis of the knee: a randomized controlled pilot trial. Clin Orthop Relat Res. 2013;471:3556-62.
44. Fuggle NR, Cooper C, Oreffo ROC, Price AJ, Kaux JF, Maheu E, et al. Alternative and complementary therapies in osteoarthritis and cartilage repair. Aging Clin Exp Res. 2020;32:547-60.

45. Reginster JY, Deroisy R, Rovati LC, Lee RL, Lejeune E, Bruyere $\mathrm{O}$, et al. Long-term effects of glucosamine sulphate on osteoarthritis progression: a randomised, placebo-controlled clinical trial. Lancet (Lond, Engl). 2001;357:251-6.

46. Saengnipanthkul S, Waikakul S, Rojanasthien S, Totemchokchyakarn K, Srinkapaibulaya A, Cheh Chin T, et al. Differentiation of patented crystalline glucosamine sulfate from other glucosamine preparations will optimize osteoarthritis treatment. Int J Rheum Dis. 2019;22:376-85.

47. Kahan A, Uebelhart D, De Vathaire F, Delmas PD, Reginster J-Y. Long-term effects of chondroitins 4 and 6 sulfate on knee osteoarthritis: the study on osteoarthritis progression prevention, a two-year, randomized, double-blind, placebo-controlled trial. Arthritis Rheum. 2009;60:524-33.

48. Harkey M, Davis J, Lu B, Price L, Ward RJ, MacKay JW, et al. Novel composite knee structure metrics of disease activity and cumulative damage predict the development of accelerated knee osteoarthritis: data from the osteoarthritis initiative. Osteoarthritis Cartil. 2019;27:S333.

49. Reginster J-YY, Reiter-Niesert $S$, Bruyère $O$, Berenbaum $F$, Brandi M-LL, Branco J, et al. Recommendations for an update of the 2010 European regulatory guideline on clinical investigation of medicinal products used in the treatment of osteoarthritis and reflections about related clinically relevant outcomes: expert consensus statement. Osteoarthritis Cartil. 2015;23:2086-93.

50. Pavelka K, Gatterova J, Olejarova M, Machacek S, Giacovelli G, Rovati LC. Glucosamine sulfate use and delay of progression of knee osteoarthritis: a 3-year, randomized, placebo-controlled, double-blind study. Arch Intern Med. 2002;162:2113-23. 